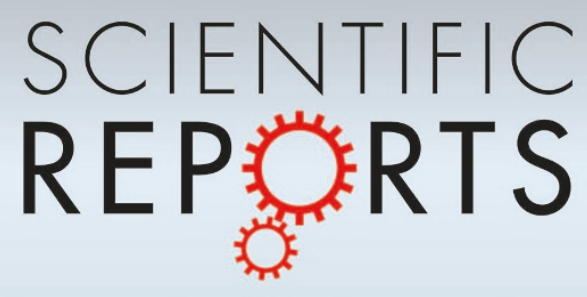

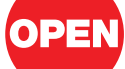

SUBJECT AREAS:

STATISTICAL PHYSICS, THERMODYNAMICS AND NONLINEAR DYNAMICS

PLASMA PHYSICS

ASTROPHYSICS

CARBON NANOTUBES AND FULLERENES

Received 4 July 2012

Accepted

1 August 2012

Published

30 August 2012

Correspondence and requests for materials should be addressed to M.M. (millmen@gmail.

com)

\section{Transition in the Equilibrium Distribution Function of Relativistic Particles}

\author{
M. Mendoza', N. A. M. Araújo', S. Succi ${ }^{2,3}$ \& H. J. Herrmann ${ }^{1,4}$ \\ ${ }^{1}$ Computational Physics for Engineering Materials, IfB, ETH Zürich, Schafmattstrasse 6, CH-8093 Zürich, Switzerland, ${ }^{2}$ Instituto per \\ le Applicazioni del Calcolo C.N.R., Via de Taurini, 1900185 , Rome, Italy, ${ }^{3}$ Freiburg Institute for Advanced Studies, Albertstrasse, \\ 19, D-79104, Freiburg, Germany, ${ }^{4}$ Departamento de Física, Universidade Federal do Ceará, Campus do Pici, $60451-970$ \\ Fortaleza, Ceará, Brazil.
}

We analyze a transition from single peaked to bimodal velocity distribution in a relativistic fluid under increasing temperature, in contrast with a non-relativistic gas, where only a monotonic broadening of the bell-shaped distribution is observed. Such transition results from the interplay between the raise in thermal energy and the constraint of maximum velocity imposed by the speed of light. We study the Bose-Einstein, the Fermi-Dirac, and the Maxwell-Jüttner distributions, and show that they all exhibit the same qualitative behavior. We characterize the nature of the transition in the framework of critical phenomena and show that it is either continuous or discontinuous, depending on the group velocity. We analyze the transition in one, two, and three dimensions, with special emphasis on twodimensions, for which a possible experiment in graphene, based on the measurement of the Johnson-Nyquist noise, is proposed.

ack in 1911, F. Jüttner derived a relativistic analogue of the Maxwell-Boltzmann equilibrium distribution for classical (non-relativistic) gases. To this purpose, he resorted to an entropy minimization procedure, subject to the relativistic energy-momentum constraints ${ }^{1}$. This top-down (macro-to-micro) derivation, left room for some debate on whether the exact form of the equilibrium distribution for relativistic particles was the one proposed by Jüttner, or rather some variant thereof ${ }^{2-4}$. Very recently, conclusive evidence for the original form proposed by Jüttner has been brought by numerical simulations of fully relativistic molecular dynamics in one, two, and three dimensions $s^{5-7}$. This is all but an academic exercise, since the Jüttner distribution is known to play a major role in the interpretation of current and future experiments in many sectors of modern physics, such as quark-gluon plasmas produced in heavy-ion collisions ${ }^{8}$, relativistic astrophysics ${ }^{9}$, distortions of the cosmic microwave background ${ }^{10}$, and lately, possibly also in the study of electron flows in graphene $e^{11,12}$.

In this article, we wish to call the attention on a special property of the Jüttner distribution, namely the fact that, under the constraint of an increasing temperature (ultrarelativistic limit $m c^{2}<k_{B} T$ ), the one-dimensional distribution develops a transition in velocity space, with the emergence of two separate peaks, moving with opposite, non zerospeeds. For two and three dimensions, it generates, in velocity space, a ring and a hollow sphere (see figure 1), respectively. Physically this corresponds to a transition between a regime where kinetic energy is mainly stored increasing the velocity and where this is done by enhancing the mass. This stands in sharp contrast with the way a non-relativistic gas at rest responds to the constraint of an increasing temperature, namely through a progressive broadening of the Gaussian shape, which enhances the high-speed population, leaving nonetheless the least-energetic, zero-speed, particles as the most probable population, since this is the one best conforming to the zero net-motion constraint. On the contrary, the transition exhibited by the Jüttner distribution, signals that, even in a gas at rest, the temperature constraint can only be met by clustering most of the particles around two oppositely moving beams, thereby depleting the zero-speed particles in the process. The above considerations readily generalize to the case of a moving gas, the main change being that the two oppositely moving beams get differently populated, the co-moving one being enhanced and the counter-moving being correspondingly depopulated. This phenomenon is quite general, and it might apply to a whole class of systems where physical signals are forced to move close to the their ultimate limiting speed. In the following, we provide mathematical details of this transition and also discuss conditions under which it could be experimentally probed in graphene experiments.

\section{Results}

Transition in the probability distribution. The probability distribution function of particle velocities in $d$ dimensional relativistic gas is described by the following single-particle distribution function (in natural units $\left(c=\hbar=e=k_{B}=m=1\right)$ and Cartesian coordinates $)^{6,13}$ : 


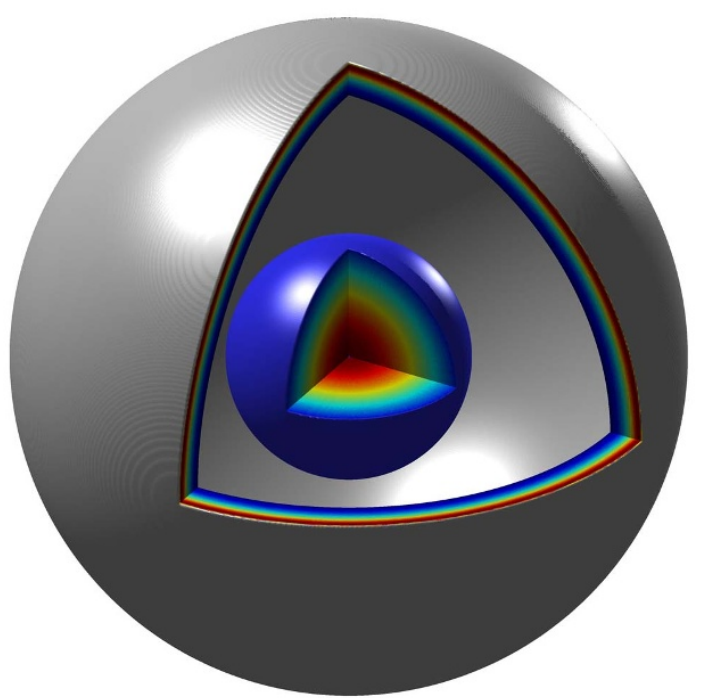

Figure 1 | Three-dimensional Maxwell-Jüttner velocity distributions, according to equation (1), for two different temperatures. The blue $(\xi=20)$ and gray $(\xi=1)$ isosurfaces stand at $1 / 3$ and $1 / 4$ of their respective maxima. Shown in the inner region of each isosurface, is the color gradient of the distribution, with red and blue colors denoting high and low concentration of particles, respectively. Note that the spheres are plotted in velocity space, so that the maximum radius for the external isosurface is close to $|\vec{v}|=\sqrt{v_{x}^{2}+v_{y}^{2}+v_{z}^{2}}=1$.

$$
f_{\lambda}(\vec{x}, \vec{v}, t)=\frac{A \gamma^{d+2}(\vec{v})}{\exp \left[\frac{1-\vec{v} \cdot \vec{U}}{T} \gamma(\vec{v}) \gamma(\vec{U})-\frac{\mu}{T}\right]+\lambda},
$$

where $\vec{v}=\left(v_{x}, v_{y}, v_{z}\right)$ is the velocity of the particles, $\gamma(\vec{v})=1 / \sqrt{1-\vec{v}^{2}}$ is the Lorentz factor, $T$ the temperature, $\vec{U}$ the group velocity, $A$ a normalization constant, $v=|\vec{v}|$, and $U=|\vec{U}|$. The subscript $\lambda$ denotes the Fermi-Dirac $(\lambda=1)$, the Bose-Einstein $(\lambda=-1)$, and the Maxwell-Jüttner $(\mu=0)$ distributions, respectively. At the moment, we will neglect the chemical potential $(\mu=0)$. The thermal behavior

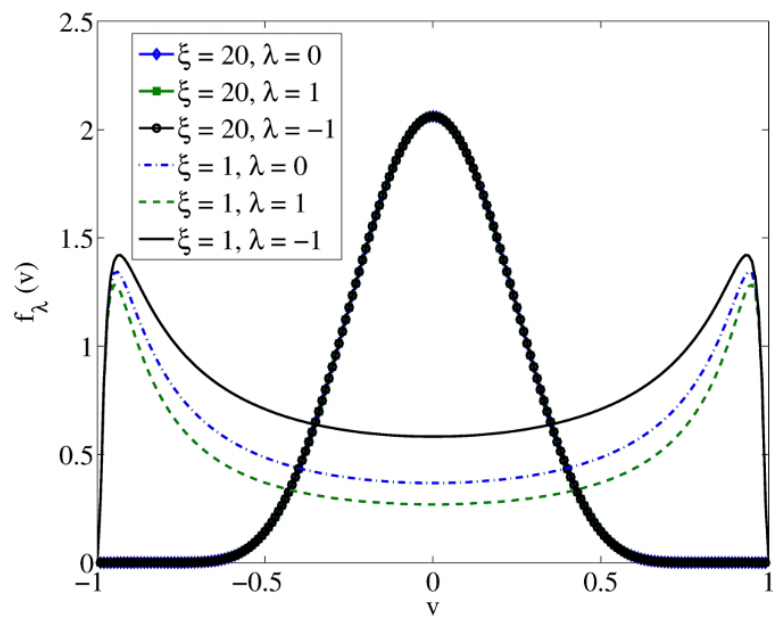

Figure $2 \mid$ Bose-Einstein $(\lambda=-1)$, Maxwell-Jüttner $(\lambda=0)$, and Fermi$\operatorname{Dirac}(\lambda=1)$ velocity distributions, for $d=1$, according to equation (1). The distributions correspond to a relativistic fluid, with group velocity $U$ $=0$ and two different temperatures $(T=1 / \xi)$, namely, $\xi=1$ and $\xi=20$. of a relativistic gas at equilibrium is best characterized by the parameter $\xi \equiv m c^{2} / k_{B} T=1 / T$, that is commonly used to differentiate between the ultra-relativistic $(\xi \gg 1)$ and the relativistic $(\xi \ll 1)$ regimes.

Under the constraint of a limiting velocity imposed by the theory of relativity, the three distributions share an interesting property in the temperature dependence. In particular, above a critical temperature $\left(T_{c}=1 / \xi_{c}\right)$, the shape of the function changes from a nearly Gaussian to bimodal (see figure 2). As a result, while below the critical temperature the majority of the particles move at speeds close to the group velocity $U$ (zero in figure 2 ), above criticality two populations of particles emerge, with a velocity distribution sharply peaked around opposite speeds, close to the speed of light. Here, we show that this change in the functional dependence of the velocity distribution can be described in the framework of a transition, which might be either discontinuous (first order) or continuous (higher order), depending on the group velocity $U$. In two and three dimensions, the same qualitative behavior is observed, where, instead of two peaks, a ring (in two dimensions) and a hollow sphere (in three dimensions, see figure 1) is obtained at higher temperatures. In both cases, at sufficiently high temperatures, the radius in velocity space reaches the value corresponding to the speed of light (see figure 1).

To characterize the transition, we introduce an order parameter defined as the distance $\Delta$ between the peaks, such that $\Delta=0$ in the single peaked distribution and $\Delta \neq 0$ in the bimodal one. Let us begin by considering the case $U=0$, and measure the order parameter dependence on the temperature, $T=1 / \xi$, as shown in figure 3(a). From this figure, we can appreciate that, below the critical temperature $T_{c}=1 / \xi_{c}$, the distribution function has only one peak, $\Delta=0$, while above $T_{c}$, a bimodal profile develops, with the order parameter growing from zero to an asymptotic value $\Delta=2$. In this limit, the width of the distribution shrinks to zero and the distribution is a superposition of two Dirac deltas, literally corresponding to a discrete fluid moving at $\pm c$. It is noteworthy that, while in the low temperature regime $\left(T<T_{c}\right)$ the dispersion in velocity around the peak increases with $T$, above the critical temperature it vanishes in the limit $T \rightarrow \infty$.

In the inset of figure 3(a), we analyze the singularity at $T=T_{c}$, namely, we plot the order parameter dependence on the rescaled control parameter $1 / \xi-1 / \xi_{c}$. A continuous transition is observed, with the order parameter being zero at the critical temperature and growing according to a power law $\Delta \sim\left(1 / \xi-1 / \xi_{c}\right)^{0.5}$ above it. This exponent corresponds to the inverse of the exponent $\delta$ in the theory of critical phenomena ${ }^{14}$. The same qualitative behavior is observed in two and three dimensions. Below, we describe the way the exponent and critical temperature can be obtained analytically, in the limit $U \rightarrow 0$.

Due to the fact that the aforementioned transition is driven by the $\gamma^{d+2}$ pre-factor in the distribution, which is symmetric around $v=0$ in velocity space, one can, without loss of generality, calculate $\Delta$ (diameter of the ring, for $d=2$, or of the hollow sphere, for $d=3$ ) along the direction $v_{x}$. In the limit $U=0$, from the calculation of the maxima of the distribution, equation (1), one obtains the trivial solution $v_{x}=0$ and two additional ones, corresponding to the solutions of the algebraic equation,

$$
(2+d) T \lambda+e^{\gamma(v) / T}((2+d) T-\gamma(v))=0 .
$$

Since $\Delta=\left|v_{x 1}-v_{x 2}\right|$, where $v_{x 1}$ and $v_{x 2}$ are the two non-zero solutions, we obtain

$$
\Delta=2 \frac{\sqrt{[2+d+W(q)]^{2} T^{2}-1}}{T(2+d+W(q))},
$$

where $q=(2+d) e^{-(2+d)} \lambda$ and $W(x)$ is the Lambert $\mathrm{W}$-function. From this equation, we can also obtain the critical temperature as, 

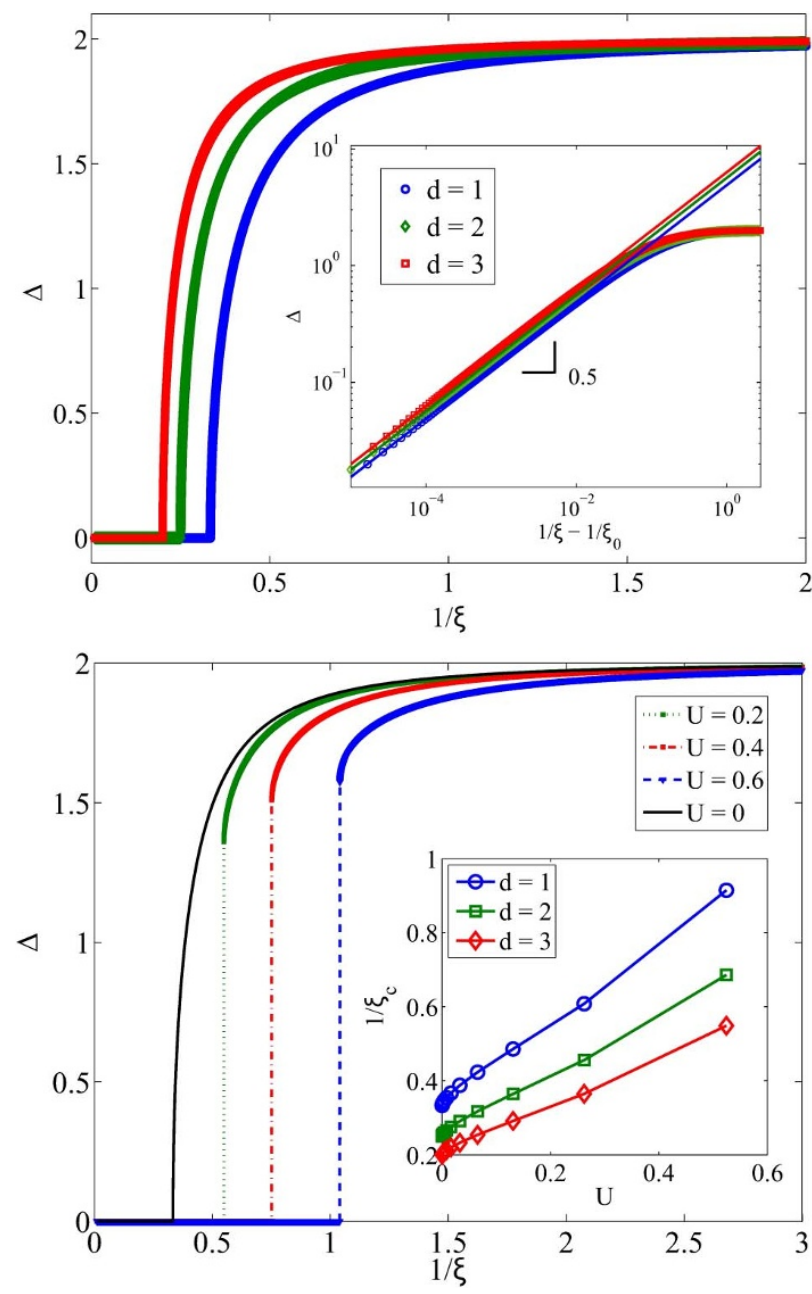

Figure 3 Temperature dependence of the order parameter. (Top panel) Temperature $(T=1 / \xi)$ dependence of the order parameter $\Delta$, defined as the distance between peaks, for the Maxwell-Jüttner distribution $(\lambda=0)$, in different spatial dimensions $d$ and group velocity $U=0$. Shown in the inset is a double-logarithmic plot of $\Delta$ as a function of the rescaled control parameter $1 / \xi-1 / \xi_{o}$ where $\xi_{0}$ stands for the transition temperature. (Bottom panel) Temperature dependence of the $\Delta$ for different group velocities. The inset shows the transition temperature $T_{c}=1 / \xi_{c}$ dependence on the group velocity $U$.

$$
T_{c}=\frac{1}{d+2+W(q)},
$$

where, in the case of the Maxwell-Jüttner distribution $(\lambda=0)$, i.e., $q=W(q)=0$, and so $T_{c}=1 /(d+2)$. In general, for this distribution, the temperature dependence of $\Delta$ is given by,

$$
\Delta=\frac{2}{\varepsilon+T_{c}} \sqrt{\varepsilon\left(\varepsilon+2 T_{c}\right)}
$$

with $\varepsilon=T-T_{c}$. For $T \gtrsim T_{c}, \Delta \sim \sqrt{8 \varepsilon / T_{c}}$, where the exponent is $1 / 2$, in excellent agreement with the numerical data in the inset of figure 3(a). This exponent depends neither on the spatial dimension, nor on $\lambda$, i.e., it is the same for the three distributions. However, the critical temperature $T_{c}$ depends on these quantities, and can be obtained analytically for each value of $d$ and $\lambda$.

Figure 3(b) shows the numerical results for the temperature dependence of $\Delta$ at different group velocities $U$, for the MaxwellJüttner distribution. For any $U \neq 0$, a jump on $\Delta$ is observed at the onset of the transition, resembling thermal first-order transitions. In figure 4 , we plot the size of the jump $\Delta_{j}$ as a function of $U$, where a power law is obtained $\Delta_{j} \sim U^{\beta}$, with $\beta=0.32 \pm 0.02$. This exponent corresponds to the order parameter exponent $\beta$ in the theory of critical phenomena ${ }^{14}$. Within the error bars, the same exponent was obtained for all combinations of $\lambda$ and $d$, suggesting that $\beta$ is independent of these two parameters. Nevertheless, as shown in the inset of figure 3(b), the transition temperature increases with $U$ and decreases with $d$.

Example in condensed matter. The above transition is not transmitted to the conserved macroscopic quantities (energymomentum) and consequently, it is not straightforwardly observed at the macroscopic level. Instead, a microscopic analysis is required. In order to experimentally detect the transition, thermal energies of the order of the rest energy of the gas particles $(\xi \sim 1)$ would be required. This could be achieved, for example, in hot electron plasmas. In particular, it is of great interest to explore whether this transition can contribute to a deeper understanding of small condensed matter systems with potential technological applications. A good candidate in this respect is graphene. Since its discovery ${ }^{15,16}$, graphene has continued to surprise scientists with an amazing series of spectacular properties, such as ultra-high electrical conductivity, ultra-low viscosity to entropy ratio, combination of exceptional structural strength and mechanical flexibility, and optical transparency. It consists of literally a single carbon monolayer and represents the first instance of a truly two-dimensional material (the "ultimate flatland"17), where electrons move like massless chiral particles and their dynamics is governed by the Dirac equation, following the dispersion relation, $E(\vec{k})=s \hbar v_{F}|\vec{k}|$, where $\vec{k}=$ $\left(k_{x}, k_{y}\right)$ is the wave vector. The constant $s= \pm 1$ distinguishes between electrons $(+)$ and holes $(-)$, and $v_{F}$ is the Fermi velocity that plays the same role in graphene as the speed of light in relativity ${ }^{18,19}$. This relation implies that carriers always move at the same Fermi speed, regardless of the Fermi energy. For simplicity, we will work only with the electronic density $(s=1)$, the extension to include holes being straightforward.

In our context, pristine graphene corresponds to the ultrarelativistic limit, where the velocity distribution function consists of two Dirac deltas at $\pm v_{F}$, so that the transition cannot be observed. However, the electronic spectrum of graphene changes depending on the substrate. For example, on $\mathrm{SiC}$ the energy spectrum presents a gap of width $2 m v_{F}^{2}=0.26 \mathrm{eV}$ and on $h$-BN (hexagonal boron nitride) a gap of $53 \mathrm{meV}^{20-22}$, and can be manipulated by constructing graphene nanoribbons, where the energy gap depends on the width of the ribbon ${ }^{23,24}$.

A gap in the spectrum of graphene corresponds to non-zero mass of the electrons, so that the dispersion relation becomes $E_{\alpha}(\vec{k})=$ $\hbar v_{F} \sqrt{\vec{k}^{2}+\alpha^{2}}$, where $\alpha=m v_{F} / \hbar$. With this dispersion relation, the velocity of the electrons can be calculated as $\vec{v}=(1 / \hbar) \partial E(\vec{k}) / \partial \vec{k}$, thus obtaining, $\vec{v}=v_{F} \vec{k} / \sqrt{\vec{k}^{2}+\alpha^{2}}$. Note that the velocity is nolonger constant and depends on the Fermi energy via $\vec{k}$. The electronic density $n$ is defined by

$$
n=\int f(\vec{k}) \frac{d^{2} k}{4 \pi^{2}},
$$

where $f(\vec{k})=\left[1+\exp \left((E(\vec{k})-\mu) / k_{B} T\right)\right]^{-1}$ is the Fermi-Dirac distribution. To calculate the density of states in velocity space, we change variables, from the wave to the velocity vector spaces, obtaining $n=\int D(\vec{v}) f(\vec{v}) d^{2} v$, where $D(\vec{v})=\alpha^{2} \gamma^{4} /(2 \pi)^{2} v_{F}$ is defined as the density of states (DoS). The Fermi-Dirac distribution function takes the form,

$$
f(\vec{v})=\frac{1}{1+\exp (\xi \gamma(v)-\eta)},
$$




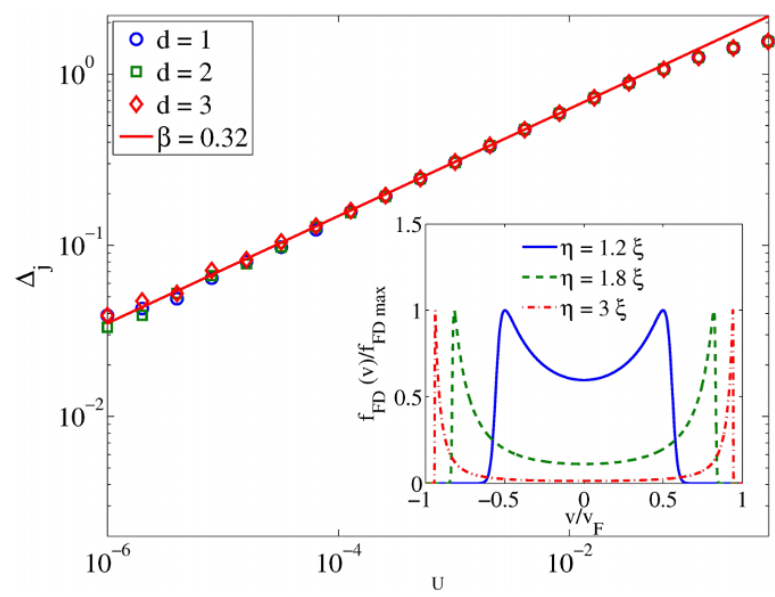

Figure $4 \mid$ Dependence on the group velocity. Size of the jump $\Delta_{j}$ as a function of the group velocity $U$ for different dimensions $d$. The quantity $\beta$ denotes the slope of the best fit. Shown in the inset, is the two-dimensional Fermi-Dirac distribution according to equation (7) for $\xi=61.6(h-\mathrm{BN}$ graphene at $5 \mathrm{~K}$ ).

with $\eta=\mu / k_{B} T$, and the parameter $\xi$ now defined as $\xi=m v_{F}^{2} / k_{B} T$. Note that the DoS tends to push all the particles moving at the Fermi velocity, while the Fermi-Dirac distribution counters this effect, given the limit imposed by the Fermi speed. At low temperature, the chemical potential can be approximated by the Fermi energy ${ }^{25}$, $E_{F}$, which can be tuned experimentally by a gate voltage for low concentration of electrons ${ }^{26}$. Since low temperatures are required, to avoid electron-phonon interactions, we characterize the transition of the velocity distribution functions by changing the Fermi energy, i.e. $\eta$, instead of the temperature. With this change in the control parameter we also have the advantage that the speed at which the Fermi-Dirac distribution attains its maximum corresponds, to a good approximation, to the maximum speed at which carriers can move (see inset of figure 4). Therefore, we expect that the transition could be observed by measuring the thermal or Johnson-Nyquist noise ${ }^{27-29}$. Let us assume that we have a typical sample of graphene on $h$ - $\mathrm{BN}$, and the electronic density is manipulated with an external gate voltage by the relation $n \simeq \kappa \eta^{30}$, where $\kappa$ is a proportionality

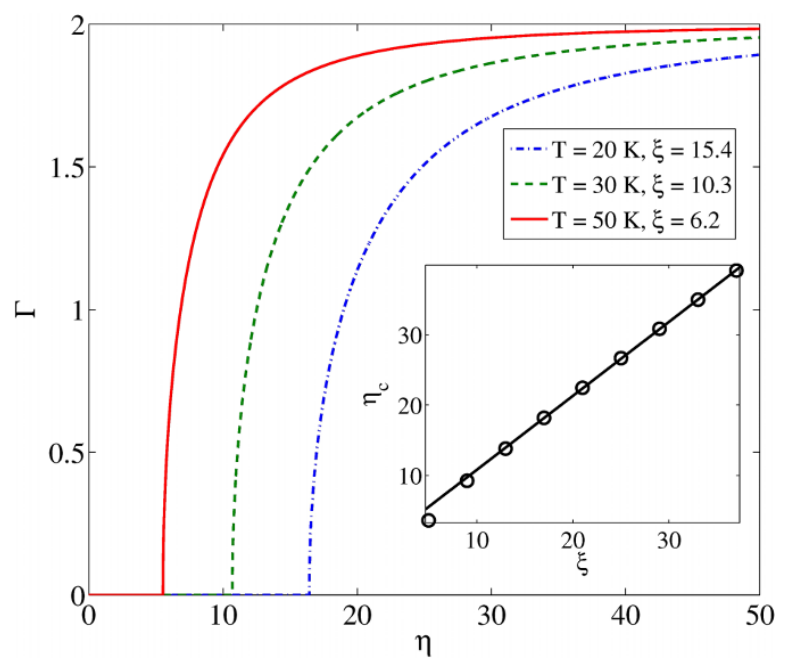

Figure $5 \mid$ Results for graphene. Dimensionless maximum amplitude for the current fluctuations $\Gamma$ for gaped graphene on a substrate $h$-BN as a function of $\eta$. The inset shows the critical $\eta_{c}$, for which the thermal noise emerges, as a function of the gap-induced mass, described by $\xi$. The solid line represents the equation $\eta_{c}=1.05 \xi+0.26$. constant that depends on the electric capacitance of the substrate and the temperature. Thus, for low carrier concentration, even if there is no drain voltage, there are current fluctuations $\delta I$ around zero due to the thermal motion of the electrons in the sample. The maximum amplitude for these fluctuations can be written as $\delta I_{\max }=e n v_{\max } l$, where $e$ and $l$ are the electric charge of the electrons and the cross section of the sample, respectively. We define the dimensionless maximum amplitude for the current fluctuations as $\Gamma=2 \delta I_{\max } / e n l v_{F}$.

For a fixed low temperature, we can observe from figure 5 that there is a critical $\eta_{c}$ above which $\Gamma$ emerges. This critical value increases with decreasing temperature. We have considered different temperatures, $T=20,30,50 \mathrm{~K}$. Note that the critical $\eta_{c}=1.05 \xi+$ 0.26 , is almost the same as $\xi$, by a proportionality constant 1.05 , and the residual is just due to the non-linear behavior of the curve, close to the critical temperature where, due to the thermal energy, the thermal noise appears, regardless of $\eta$. Note that this expression is tantamount to stating that the Fermi level must be higher than half of the gap energy in order to have electrons in the conduction band. The exponent of the continuous transition is also 0.5 , and is independent of the temperature of the sample in the regime of low temperatures.

Some transport properties in graphene also depend on the velocity instead of the momentum, e.g. saturation current in ballistic regime $e^{31,32}$. Since electrons in pristine graphene always move at the Fermi speed, which is constant, the presence of a bias potential will mainly increase the concentration of carriers and change the direction of motion of the carriers (not their speed). Consequently, the saturation current is achieved when most of the carriers move along the direction of the electric field. When the graphene sample presents a gap, for low bias voltage, the maximum velocity achieved by the carriers is mainly dominated by the Fermi level, following the curve in the inset of Fig. 5. Therefore, the quantity $\Delta / 2$ (with $\Delta$ the separation between peaks for the velocity distribution of carriers) plays an important role in the determination of the saturation electrical current of the sample. This shows that it is plausible to expect that the transition in the Fermi-Dirac distribution discussed in this article might help understanding (some of) the spectacular properties of graphene. Furthermore, with the aim of building graphene transistors at the nanoscale, the study of the transport properties of nanoribbons (GNR) ${ }^{23,24}$ has become very popular, where understanding the individual carrier dynamics might be relevant ${ }^{33}$.

\section{Discussion}

Summarizing, we have shown that the equilibrium distribution for relativistic particles, no matter whether classical or quantum, exhibits a transition as the temperature is brought close to the rest energy (ultra-relativistic limit). This transition is the organized response of the distribution of particles to the constraint of an increasing temperature, compatibly with the existence of a limiting speed for the propagation of physical signals. We have also discussed conditions under which such transition could potentially be detected in current and future graphene experiments. In the case of graphene, we have found that the transition takes place, not only by increasing the temperature, but also by increasing the Fermi energy at constant low temperature. In principle, we expect the same qualitative behavior in any system in which an equivalent of the Fermi energy (or chemical potential) could be defined.

\section{Methods}

Results for $U=0$ and $\mu=0$ were obtained by algebraically solving the distribution functions and their maxima, while for the other cases the maxima were computed numerically.

1. Jüttner, F. Das Maxwellsche Gesetz der Geschwindigkeitsverteilung in der Relativtheorie. Annalen der Physik 339, 856-882 (1911).

2. Lehmann, E. Covariant equilibrium statistical mechanics. J. Math. Phys. 47, 023303 (2006). 
3. Dunkel, J. \& Hänggi, P. One-dimensional non-relativistic and relativistic brownian motions: a microscopic collision model. Physica A 374, 559-572 (2007).

4. Cubero, D. \& Dunkel, J. Stationarity, ergodicity, and entropy in relativistic systems. EPL (Europhysics Letters) 87, 30005 (2009).

5. Cubero, D., Casado-Pascual, J., Dunkel, J., Talkner, P. \& Hänggi, P. Thermal equilibrium and statistical thermometers in special relativity. Phys. Rev. Lett. 99, 170601 (2007).

6. Montakhab, A., Ghodrat, M. \& Barati, M. Statistical thermodynamics of a two-dimensional relativistic gas. Phys. Rev. E 79, 031124 (2009).

7. Dunkel, J., Hänggi, P. \& Hilbert, S. Non-local observables and lightcone-averaging in relativistic thermodynamics. Nat. Phys. 5, 741-747 (2009).

8. Shuryak, E. Why does the quark-gluon plasma at rhic behave as a nearly ideal fluid? Progress in Particle and Nuclear Physics 53, 273-303 (2004).

9. Soderberg, A. M. et al. A relativistic type lbc supernova without a detected $\gamma$-ray burst. Nature 463, 513-515 (2010).

10. Prokhorov, D., Colafrancesco, S., Akahori, T., Yoshikawa, K., Nagataki, S. \& Seon, K.-I. Can electron distribution functions be derived through the SunyaevZel'dovich effect? Astron. Astrophys. 529, A39 (2011).

11. Müller, M. \& Sachdev, S. Collective cyclotron motion of the relativistic plasma in graphene. Phys. Rev. B 78, 115419 (2008).

12. Mendoza, M., Herrmann, H. J. \& Succi, S. Preturbulent regimes in graphene flow. Phys. Rev. Lett. 106, 156601 (2011).

13. Cercignani, C. \& Kremer, G. M. The Relativistic Boltzmann Equation: Theory and Applications. Birkhauser, Berlin (2002).

14. Stanley, H. E. Introduction to Phase Transitions and Critical Phenomena. Oxford Science Publications, New York (1971).

15. Novoselov, K. et al. Two-dimensional gas of massless dirac fermions in graphene. Nature 438, 197-200 (2005).

16. Novoselov, K. S. et al. Electric Field Effect in Atomically Thin Carbon Films. Science 306, 666-669 (2004).

17. Geim, A. K. \& MacDonald, A. H. Graphene: Exploring carbon flatland. Phys Today 60, 35-41 (2007).

18. Castro Neto, A. H., Guinea, F., Peres, N. M. R., Novoselov, K. S. \& Geim, A. K. The electronic properties of graphene. Rev. Mod. Phys. 81, 109-162 (2009)

19. Peres, N. M. R. Colloquium: The transport properties of graphene: An introduction. Rev. Mod. Phys. 82, 2673-2700 (2010).

20. Zhou, S. Y. et al. Substrate-induced bandgap opening in epitaxial graphene. Nat. Mater. 6, 770-775 (2007).

21. Pereira, V. M., Kotov, V. N. \& Castro Neto, A. H. Supercritical coulomb impurities in gapped graphene. Phys. Rev. B 78, 085101 (2008).

22. Giovannetti, G., Khomyakov, P. A., Brocks, G., Kelly, P. J. \& van den Brink, J. Substrateinduced band gap in graphene on hexagonal boron nitride: $A b$ initio density functional calculations. Phys. Rev. B 76, 073103 (2007).

23. Han, M. Y., Özyilmaz, B., Zhang, Y. \& Kim, P. Energy band-gap engineering of graphene nanoribbons. Phys. Rev. Lett. 98, 206805 (2007).
24. Barone, V., Hod, O. \& Scuseria, G. E. Electronic structure and stability of semiconducting graphene nanoribbons. Nano Letters 6, 2748-2754 (2006).

25. Ashcroft, N. \& Mermin, N. Solid State Physics. Thomson Learning, London (1976).

26. Das Sarma, S., Adam, S., Hwang, E. H. \& Rossi, E. Electronic transport in twodimensional graphene. Rev. Mod. Phys. 83, 407-470 (2011).

27. Johnson, J. B. Thermal agitation of electricity in conductors. Phys. Rev. 32, 97-109 (1928).

28. Nyquist, H. Thermal agitation of electric charge in conductors. newblock Phys. Rev. 32, 110-113 (1928).

29. Bulashenko, O. M. Johnson-nyquist noise spectrum for $2 \mathrm{~d}$ electron gas in a narrow channel. AIP Conference Proceedings 285, 23-26 (1993).

30. Meric, I., Dean, C., Young, A., Hone, J., Kim, P. \& Shepard, K. Graphene fieldeffect transistors based on boron nitride gate dielectrics. In Electron Devices Meeting (IEDM), 2010 IEEE International, 23.2.1-23.2.4 (2010).

31. Saad, I., Tan, M. L., Hii, I. H., Ismail, R. \& Arora, V. K. Ballistic mobility and saturation velocity in low-dimensional nanostructures. Microelectronics Journal 40, 540-542 (2009).

32. Amin, N. A., Ahmadi, M. T., Johari, Z., Webb, J. F., Mousavi, S. M. \& Ismail, R. Drift velocity and mobility of a graphene nanoribbon in a high magnitude electric field. AIP Conference Proceedings 1337, 177-179 (2011).

33. Pototsky, A., Marchesoni, F., Kusmartsev, F. V., Hänggi, P. \& Savel'ev, S. E. Relativistic brownian motion on a graphene chip. arXiv:1103.0945v3.

\section{Acknowledgments}

We acknowledge the Brazilian institute INCT-SC.

\section{Author contributions}

All authors conceived and designed the research, analyzed the data, worked out the theory, and wrote the manuscript.

\section{Additional information}

Competing financial interests: The authors declare no competing financial interests.

License: This work is licensed under a Creative Commons

Attribution-NonCommercial-NoDerivative Works 3.0 Unported License. To view a copy of this license, visit http://creativecommons.org/licenses/by-nc-nd/3.0/

How to cite this article: Mendoza, M., Araújo, N.A.M., Succi, S. \& Herrmann, H.J. Transition in the Equilibrium Distribution Function of Relativistic Particles. Sci. Rep. 2, 611; DOI:10.1038/srep00611 (2012) 